PALABRAS CLAVE

Tributación

Impuesto a la renta

Reforma tributaria

Distribución del ingreso

Medición

Estudios de casos

Guatemala

Santiago Díaz de Sarralde

Profesor titular del Departamento de

Economía Aplicada II, Universidad Rey

Juan Carlos. Campus de Vicálvaro

- santiago.diazsarralde@correo.aeat.es

Carlos Garcimartín

Profesor titular del Departamento de Economía Aplicada II, Universidad Rey Juan Carlos. Campus de Vicálvaro

œ carlos.garcimartin@urjc.es

Jesús Ruiz-Huerta

Catedrático del Departamento de Economía Aplicada II, Universidad Rey Juan Carlos. Campus de Vicálvaro

œ jesus.ruizhuerta@urjc.es
REVISTA CEPAL 102 DICIEMBRE 2010

\section{La paradoja de la progresividad en países de baja tributación: el impuesto a la renta en Guatemala}

\author{
Santiago Diaz de Sarralde, Carlos Garcimartin y \\ Jesús Ruiz-Huerta
}

$\mathrm{L}$

os índices de Kakwani y Reynolds-Smolensky se emplean para analizar las consecuencias de las reformas fiscales en la progresividad y capacidad redistributiva de los impuestos. No obstante, su interpretación en términos normativos solo resulta pertinente en reformas en que la recaudación permanece constante. Dado que este no suele ser el caso en las reformas reales, es habitual descomponer el índice de Reynolds-Smolensky en cambios en la tasa media del impuesto y cambios en el índice de Kakwani. En este trabajo se intenta mostrar que esta descomposición presenta serios inconvenientes, que se agravan en países de bajos ingresos tributarios. Para contribuir a la solución de estos problemas se propone una serie de indicadores alternativos basados en los índices tradicionales, que permiten analizar los efectos en la redistribución y progresividad en reformas con variaciones en la recaudación. Mediante estos indicadores se examinan hipotéticas reformas en el impuesto sobre la renta de Guatemala. 


\section{I}

\section{Introducción}

$\mathrm{Al}$ analizar los efectos de una reforma fiscal en la progresividad y capacidad redistributiva, resulta habitual en la literatura utilizar las variaciones de los índices de Kakwani (1977) y de Reynolds-Smolensky (1977). Sin embargo, estos índices pueden no resultar apropiados para efectuar valoraciones normativas en presencia de reformas fiscales que suponen cambios significativos en la recaudación. Para solucionar este problema se han seguido tradicionalmente dos vías. La primera de ellas consiste en comparar las distribuciones de la renta después de impuestos mediante curvas generalizadas de concentración (curvas de Lorenz generalizadas). En la segunda vía se aprovecha la descomposición del índice de Reynolds-Smolensky, distinguiendo en la variación de la capacidad redistributiva del impuesto aquella parte causada por los cambios en la tasa media efectiva de aquella otra originada por modificaciones en la progresividad.

No obstante, como se intentará poner de manifiesto a lo largo de las siguientes páginas, esta neta separación entre los efectos de una reforma en la tasa media y la progresividad es discutible, ya que puede ocurrir que aparezca como regresiva una reforma que no solo aumenta la capacidad redistributiva de los impuestos, sino que además incrementa las diferencias entre las cuotas pagadas por los contribuyentes de rentas alta y baja. Este problema es más grave, si cabe, en países de baja tributación, donde además es habitual que los niveles de evasión sean elevados y los sistemas impositivos poco equitativos. De este modo, reformas necesarias para aumentar los recursos disponibles del sector público pueden no efectuarse bajo el argumento de ser regresivas, cuando en realidad pueden suponer tanto una mayor capacidad de redistribución de la renta como una marcada diferencia entre los impuestos pagados por los individuos de rentas alta y baja. De hecho, en el caso particular de Guatemala, uno de los países de menor tributación de América Latina, se subrayará cómo dos medidas que incrementan significativamente la capacidad de recaudación del impuesto sobre la renta pueden aparecer como regresivas si se emplean los indicadores habituales. Sin embargo, esta regresividad es aparente y posiblemente contraria a la percepción subjetiva que los ciudadanos tienen sobre la progresividad.

Por consiguiente, es necesario desarrollar otros mecanismos capaces de evaluar reformas fiscales que generen, como suele ocurrir, cambios en la recaudación y complementen la información aportada por los indicadores tradicionales. Para ello se emplearán dos conceptos que sí son separables: el nivel impositivo y las distancias entre rentas netas o cuotas tributarias. A partir de esta separación, mediante lo que se denominará efectos nivel y distancia, el objetivo es contar con otra herramienta de análisis que permita valorar los diseños de estructuras fiscales con consecuencias recaudatorias diferentes, tanto en términos de progresividad como de capacidad redistributiva, estimando en ambos casos las contribuciones de cada uno de los mencionados efectos.

En las secciones II y III de este trabajo se dará cuenta de las principales debilidades que, a juicio de los autores, presentan los índices habituales para valorar este tipo de reformas. En la sección IV se formulará la propuesta y, por último, en la sección $\mathrm{V}$ se emplearán los indicadores desarrollados para evaluar dos reformas hipotéticas en el impuesto sobre la renta de Guatemala. 


\section{II}

\section{Instrumentos de medición: desigualdad, progresividad y redistribución}

Es obvio que cualquier análisis de los efectos redistributivos de las reformas fiscales requiere, en primer lugar, un instrumento que sintetice la distribución de la renta en las diversas situaciones. Una herramienta muy extendida empleada para este fin es la llamada curva de Lorenz $(L x)$, que proporciona un sistema normalizado de medición de los porcentajes de participación de los diversos individuos en la renta total. Derivado de la curva de Lorenz y con el propósito de sintetizar en un único indicador la desigualdad relativa, es habitual emplear el coeficiente de Gini $\left(G_{x}\right)$. Gráficamente, como es bien sabido, este coeficiente mide la relación entre el área existente entre la curva de Lorenz y la diagonal y el área total bajo la diagonal, siendo su expresión matemática para distribuciones discretas de renta:

$$
G_{x}=\frac{\sum_{i=1}^{N} \sum_{j=1}^{N}\left|x_{i}-x_{j}\right|}{2 N^{2} \mu}
$$

donde $\mu$ representa la renta media, $x_{i}$ y $x_{j}$ las rentas de los sujetos $i$ y $j$, respectivamente, y $N$ la población. Por tanto, el coeficiente de Gini expresa la diferencia media entre pares de rentas dividida por el doble de la renta media, pudiendo situarse entre los valores 0 (igualdad absoluta) y 1 (un solo perceptor recibe toda la renta del conjunto). Tal como sucede con la curva de Lorenz, este coeficiente evidencia la desigualdad relativa de un conjunto de rentas, no las desigualdades absolutas, por lo que es difícil su interpretación en términos de bienestar cuando el nivel medio de las rentas difiere entre dos poblaciones.

Tal como los indicadores anteriores pueden ser útiles a la hora de comparar distintas distribuciones tanto en el tiempo como en el espacio, también de ellos pueden derivarse reformulaciones destinadas a comparar los cambios en la distribución de la renta introducidos por el sistema fiscal. Así, si para simplificar suponemos que todas las unidades de población con la misma renta soportan exactamente la misma presión tributaria, ${ }^{1}$ es

\footnotetext{
${ }^{1}$ De acuerdo con la denominación empleada por la CEPAL, por presión tributaria entendemos la cuantía de impuestos pagados en relación con la renta.
}

decir, que la cantidad de impuestos pagados solo depende de la renta, es posible representar la distribución de dichas cantidades mediante una técnica similar a la descrita en las curvas de Lorenz, obteniendo la curva de concentración de los impuestos $(L t)$. Vinculado a esta se encuentra el coeficiente de concentración $C t$, índice análogo al de Gini. De igual forma podríamos obtener la curva de concentración de las rentas después de impuestos $(L x-t)$ y su correspondiente coeficiente de concentración $(C x-t)$ (si por simplicidad suponemos que no se producen reordenaciones, $C x-t=G x-t$ ) (véase el gráfico 1).

En particular, el índice de concentración de las cuotas impositivas sería:

$$
C_{t}=\frac{\sum_{i=1}^{N} \sum_{j=1}^{N}\left|t\left(x_{i}\right)-t\left(x_{j}\right)\right|}{2 N^{2} \mu t} ; 0 \leq t \leq 1
$$

GRÁFICO

Curva de Lorenz de la renta y curvas de concentración de cuotas y de rentas netas

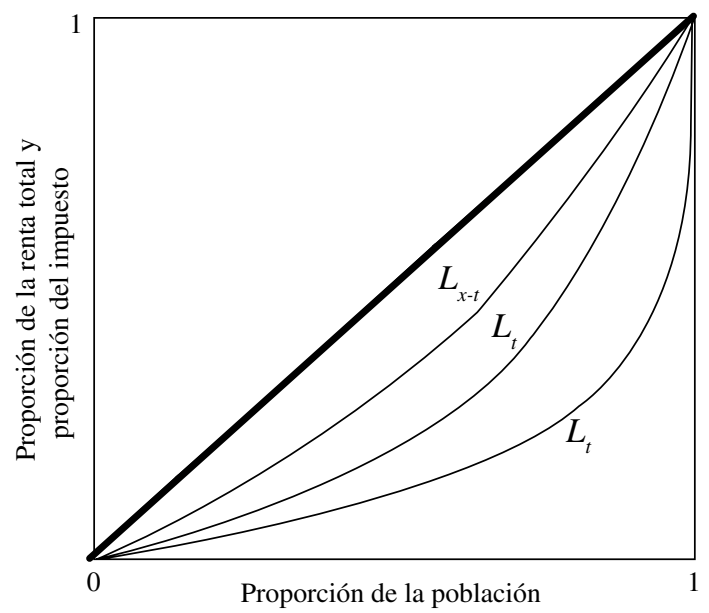

Fuente: elaboración propia.

$L_{x}: \quad$ Curva de concentración de la renta antes de impuestos.

$L_{x-t}:$ Curva de concentración de la renta después de impuestos.

$L_{t}^{x-l} \quad$ Curva de concentración de las cuotas. 
donde $t\left(x_{i, j}\right)$ representa la cuota de los contribuyentes $i, j$, mientras que $t$ es la tasa media efectiva. Por su parte, el coeficiente de concentración de la renta después de impuestos sería

$$
C_{x-t}=\frac{\sum_{i=1}^{N} \sum_{j=1}^{N}\left|\left(x_{i}-t\left(x_{i}\right)\right)-\left(x_{j}-t\left(x_{j}\right)\right)\right|}{2 N^{2} \mu(1-t)}
$$

En el caso de que un impuesto sea progresivo, las cuotas se desvían sistemáticamente de la proporcionalidad respecto de la renta antes de impuestos. Esta distribución más desigual de las cuotas que de las rentas implica que la curva de concentración de las cuotas se encuentra más alejada de la diagonal que la curva de Lorenz de la renta antes de impuestos, es decir, usando la notación habitual $L x>L t$. Dado que $L x$ no solo representa la curva de Lorenz de la renta antes de impuestos, sino también la curva de concentración de las cuotas impositivas que se obtendría con un impuesto proporcional de igual capacidad recaudatoria, es posible interpretar la separación entre dichas curvas $(L x-L t)$ como una medida de la desviación de la proporcionalidad del impuesto. Este es precisamente el objetivo del índice de Kakwani de desviación de la proporcionalidad $(K)$, habitualmente utilizado en la literatura, que mide el doble del área existente entre la curva de Lorenz de la renta antes de impuestos y la curva de concentración de las cuotas del impuesto. En otras palabras, representa la diferencia entre el coeficiente de concentración del impuesto y el coeficiente de Gini de la renta antes de impuestos:

$$
K=C t-G x
$$

A su vez, un impuesto progresivo también generaría cambios en la distribución de la renta existente antes y después de su pago. Es habitual cuantificar este efecto redistributivo mediante la distancia entre las curvas de Lorenz antes y después del impuesto $(L x-t-L x)$, pues en el caso de una imposición proporcional ambas curvas serían iguales. Esta distancia puede sintetizarse por medio del conocido índice de Reynolds-Smolensky $(R S)$ :

$$
R S=G x-C x-t
$$

Resulta obvio que la desviación de la proporcionalidad y el efecto redistributivo son dos fenómenos estrechamente relacionados. De hecho, los índices correspondientes se hallan vinculados por la siguiente igualdad: ${ }^{2}$

$$
R S=\frac{t}{1-t} K
$$

De este modo, el efecto redistributivo vendría determinado por la desviación respecto de la proporcionalidad y por el nivel del impuesto. En otras palabras, el efecto redistributivo dependería no solo de la progresividad del impuesto, sino también de su nivel.

\footnotetext{
${ }^{2}$ Una explicación de esta expresión puede verse en Lambert (2001, pág. 206 y siguientes).
} 


\section{III}

\section{Progresividad, redistribución y reformas fiscales}

Los índices anteriormente descritos son los que más se utilizan a la hora de analizar las consecuencias de una determinada reforma fiscal en la progresividad y redistribución de la renta. ${ }^{3}$ Sin embargo, pueden no resultar apropiados cuando se emplean en el análisis de reformas fiscales que suponen cambios significativos en la recaudación. Precisamente, el objetivo del presente trabajo consiste en desarrollar unos indicadores que contribuyan a subsanar algunos de los inconvenientes de dichos índices.

Como se mencionó anteriormente, la curva de Lorenz se basa en la comparación de una distribución de la renta con respecto a la proporcionalidad, mientras que el coeficiente de Gini derivado de ella mide la suma de las diferencias entre pares de renta en relación con la renta media. Por tanto, en ambos casos se trata de comparaciones relativas en que no importan los niveles, sino las proporciones. Ahora bien, en el momento de establecer comparaciones de dichos indicadores en situaciones donde los niveles varían significativamente en el tiempo o en el espacio, en la mayoría de las investigaciones se suele poner de manifiesto la limitación de estos instrumentos para ofrecer valoraciones en términos de bienestar. ${ }^{4}$ Para superar estos problemas se utilizan con frecuencia los desarrollos basados en los trabajos de Atkinson (1970) y Shorrocks (1983), por medio de la curva de Lorenz generalizada, es decir, la curva de Lorenz ordinaria multiplicada por la renta media. De este modo, pueden compararse no solo distribuciones, sino también niveles, lo que resulta más apropiado para valorar normativamente los cambios o diferencias en la distribución de la renta en una amplia gama de situaciones, si bien persisten algunos casos en que sigue siendo difícil emitir un juicio en términos de bienestar.

Sin embargo, cuando se valora una reforma fiscal las reticencias parecen ser menores, siendo muy habitual utilizar las curvas de Lorenz y de concentración —así como los índices de desigualdad, progresividad

\footnotetext{
${ }^{3}$ Son innumerables los trabajos empíricos realizados, tanto a nivel nacional como internacional, donde se emplean dichos índices.

${ }^{4}$ De forma similar a lo que ocurre cuando las curvas de Lorenz se cruzan. Véase Lambert (2001, pág. 44 y siguientes).
}

y redistribución vinculados a ellas - para comparar sus valores antes y después de la reforma, obteniendo consecuencias "normativas" sobre el diseño de la reforma en virtud de las diferencias observadas. Tales comparaciones y juicios normativos son correctos si la recaudación total no se ve alterada. En caso contrario, la obtención, por ejemplo, de un resultado de mayor progresividad o de una mayor redistribución tras la reforma no tiene por sî misma contenido normativo alguno, pues la "bondad" de la progresividad o del efecto redistributivo de un impuesto solo se puede justificar mediante la comparación con un impuesto proporcional que dé lugar a la misma recaudación.

Para resolver este problema se han utilizado dos vías en los trabajos de evaluación de reformas fiscales. La primera de ellas consiste en comparar las distribuciones de las rentas después de impuestos mediante curvas generalizadas de Lorenz. En nuestra opinión, esta argumentación es cuestionable. Así, puede imaginarse una reforma fiscal que deje inalterada la curva de Lorenz tras la aplicación de impuestos al tiempo que estos disminuyen. En ese caso, utilizar las curvas generalizadas supondría concluir que el bienestar ha aumentado. Sin embargo, se trata de una conclusión muy discutible, pues aceptarla implicaría asumir que cualquier gasto público supone una menor utilidad que la del gasto privado y esa, y no otra, sería la razón del aparente incremento del bienestar. El efecto sería similar al que se produciría al construir curvas de Lorenz después de realizar un gasto en un bien cualquiera. $\mathrm{Si}$ el gasto en este bien aumentara proporcionalmente debido a un cambio en las preferencias de la población, las curvas generalizadas nos dirían que el bienestar ha disminuido, puesto que hay menos renta disponible para el resto de bienes, cuando en realidad lo único que se ha producido es un cambio en las preferencias. Por otra parte, puede suceder que las variaciones en recaudación se compensen con otros impuestos, en cuyo caso sus efectos deberían igualmente considerarse. En última instancia, la justificación para una comparación mediante el empleo de curvas de Lorenz generalizadas reside en el hecho de que no solo la distribución importa, sino también el nivel absoluto de renta media. 
Empero, esto no parece apropiado en una reforma fiscal, pues la renta media del país en cuestión antes de impuestos no tiene porqué verse afectada por dicha reforma al menos en el corto plazo, y si ello sucede el efecto debería hacerse explícito. ${ }^{5}$

La segunda vía para valorar los efectos de una reforma fiscal que haga variar la recaudación consiste en aprovechar la descomposición del índice de Reynolds-Smolenski $(R S)$ señalada en la sección anterior, distinguiendo la variación en la capacidad redistributiva del impuesto causada por los cambios en su tasa media efectiva $(t / 1-t)$ de la variación generada por los cambios en la progresividad $(K)$. Una disminución (aumento) del nivel del impuesto a causa de una merma (incremento) de $t$ siempre tendría un efecto negativo (positivo) en $R S$, cuando el impuesto es progresivo. E igual efecto tendría una reducción (aumento) de la progresividad, medida por $K$. Así, ante una reforma que disminuya $t$, solo cabe esperar que el acrecentamiento de la progresividad sea suficiente como para compensar el cambio en el nivel impositivo.

La utilidad de este procedimiento estriba en que parece permitir una valoración separada de lo ocurrido en términos de nivel de recaudación y de progresividad, haciendo "explícito" el intercambio. De este modo sería posible, por ejemplo, valorar positivamente el aumento de la progresividad, medida a través de $K$ y atribuible al diseño de la reforma, y responsabilizar de la disminución (o menor incremento) de la redistribución únicamente a la cuantía de la rebaja fiscal.

Sin embargo, en el caso habitual de reformas fiscales que alteran la recaudación, esta separación en la atribución de responsabilidades entre el diseño de

\footnotetext{
${ }^{5}$ Un cambio impositivo a la baja no necesariamente ha de traducirse en una expansión económica inmediata. En todo caso, no resulta fácil cuantificar su repercusión en contextos económicos en que otras variables desempeñan un papel relevante.
}

la reforma y la cuantía de su efecto recaudatorio no es correcta, dado que la alteración de la recaudación no solo modifica la escala de medición, sino también la distribución de la presión tributaria. Solo una reforma fiscal que alterase en igual proporción todas las cuotas impositivas dejaría invariante el indicador de progresividad $(K)$, de modo que toda la variación de la capacidad redistributiva $(R S)$ podría atribuirse a cambios en la capacidad recaudatoria del impuesto. En este sentido puede decirse que, pese a que la medición de la progresividad es en sí misma invariante con respecto a la escala, su variación en caso de una reforma depende en última instancia de las mismas decisiones que hacen cambiar el nivel impositivo. Así, dado que $R S$ puede expresarse como:

$$
\begin{aligned}
& R S=\frac{t}{1-t} K=\frac{t}{1-t}\left(C_{t}-G_{x}\right)=\frac{t}{1-t} \\
& \left(\frac{\sum_{i=1}^{N} \sum_{j=1}^{N}\left|t\left(x_{i}\right)-t\left(x_{j}\right)\right|}{2 N^{2} \mu t}-G_{x}\right)
\end{aligned}
$$

se pone claramente de manifiesto que la progresividad no es separable del nivel impositivo, pues este afecta a aquella, medida por $K$, a través de cambios en el denominador de la expresión (7), mientras que la distribución individual del cambio en el nivel afecta a $K$ alterando su numerador, y solo en el caso de una alteración proporcional de todas las cuotas impositivas estas variaciones dejarían constante el cociente. Es decir, nivel y progresividad no son conceptos separables a la hora de evaluar el diseño de una reforma fiscal y sus efectos, por lo que el uso de los indicadores derivados de ellos para evaluar reformas fiscales puede dar lugar a errores de interpretación. 


\section{IV}

\section{La evaluación de las reformas fiscales: una propuesta alternativa basada en los efectos nivel y distancia}

\section{Reformas fiscales y redistribución}

En el sentido expuesto en la sección anterior, nivel impositivo y progresividad no son conceptos separables. En cambio sí se pueden separar los cambios en el nivel impositivo y en las distancias entre rentas netas o cuotas, lo que nos puede permitir un análisis más apropiado de las reformas fiscales en que se produzcan cambios en la recaudación. Esta es la idea fundamental en que se basa nuestra propuesta y, para desarrollarla, es oportuno comenzar recordando que una reforma aumentará el efecto redistributivo del impuesto si el índice de ReynoldsSmolenski tras ella $\left(R S^{\prime}\right)$ es superior al índice previo. En caso contrario $\left(R S^{\prime}-R S<0\right)$, la reforma reducirá el efecto redistributivo, resultando neutral si $R S^{\prime}=R S$.

La propuesta de este trabajo consiste precisamente en descomponer la variación del índice de ReynoldsSmolenski con el objeto de separar los cambios en las distancias entre rentas netas de los cambios en la tasa media. Es decir, si partimos de la expresión:

$$
R S^{\prime}-R S=\left(G^{\prime} x-C^{\prime}{ }_{x-t}\right)-\left(G x-C_{x-t}\right)
$$

y, para simplificar, suponemos que el coeficiente de Gini antes y después de la reforma no ha cambiado, ${ }^{6}$ es decir, $G^{\prime} x=G x$, entonces

$$
\begin{aligned}
& R S^{\prime}-R S=C_{x-t}-C^{\prime}{ }_{x-t}=\frac{\sum_{i=1}^{N} \sum_{j=1}^{N}\left|\left(x_{i}-t\left(x_{i}\right)\right)-\left(x_{j}-t\left(x_{j}\right)\right)\right|}{2 N^{2} \mu(1-t)}- \\
& -\frac{\sum_{i=1}^{N} \sum_{j=1}^{N}\left|\left(x_{i}-t^{\prime}\left(x_{i}\right)\right)-\left(x_{j}-t^{\prime}\left(x_{j}\right)\right)\right|}{2 N^{2} \mu\left(1-t^{\prime}\right)}
\end{aligned}
$$

\footnotetext{
${ }^{6}$ De hecho, en los ejercicios empíricos de evaluación de las reformas fiscales se suele utilizar una misma distribución de la renta, a la que se aplican las diferentes estructuras fiscales para comparar los efectos de la reforma.
}

donde el supraíndice (') representa el valor de la variable correspondiente tras la reforma. Esta expresión puede descomponerse como sigue:

$$
\begin{aligned}
& R S^{\prime}-R S=C_{x-t}-C^{\prime}{ }_{x-t}=\frac{\sum_{i=1}^{N} \sum_{j=1}^{N}\left|\left(x_{i}-t\left(x_{i}\right)\right)-\left(x_{j}-t\left(x_{j}\right)\right)\right|}{2 N^{2} \mu(1-t)}- \\
& -\frac{\sum_{i=1}^{N} \sum_{j=1}^{N}\left|\left(x_{i}-t^{\prime}\left(x_{i}\right)\right)-\left(x_{j}-t^{\prime}\left(x_{j}\right)\right)\right|}{2 N^{2} \mu\left(1-t^{\prime}\right)}+\frac{\sum_{i=1}^{N} \sum_{j=1}^{N}\left|\left(x_{i}-t\left(x_{i}\right)\right)-\left(x_{j}-t\left(x_{j}\right)\right)\right|}{2 N^{2} \mu\left(1-t^{\prime}\right)}- \\
& -\frac{\sum_{i=1}^{N} \sum_{j=1}^{N}\left|\left(x_{i}-t\left(x_{i}\right)\right)-\left(x_{j}-t\left(x_{j}\right)\right)\right|}{2 N^{2} \mu\left(1-t^{\prime}\right)}
\end{aligned}
$$

$\mathrm{y}$, por tanto,

$$
\begin{aligned}
& R S^{\prime}-R S=\frac{\sum_{i=1}^{N} \sum_{j=1}^{N}\left|\left(x_{i}-t\left(x_{i}\right)\right)-\left(x_{j}-t\left(x_{j}\right)\right)\right|}{2 N^{2} \mu(1-t)}\left(1-\frac{1-t}{1-t^{\prime}}\right)+ \\
& +\frac{\sum_{i=1}^{N} \sum_{j=1}^{N}\left|\left(x_{i}-t\left(x_{i}\right)\right)-\left(x_{j}-t\left(x_{j}\right)\right)\right|-\sum_{i=1}^{N} \sum_{j=1}^{N}\left|\left(x_{i}-t^{\prime}\left(x_{i}\right)\right)-\left(x_{j}-t^{\prime}\left(x_{j}\right)\right)\right|}{2 N^{2} \mu\left(1-t^{\prime}\right)}
\end{aligned}
$$

A objeto de mostrar más claramente el significado de esta expresión, resulta oportuno denominar $\beta$ a la tasa de variación de la renta neta media tras la aplicación de impuestos, $D$ a la suma de las distancias entre rentas netas previa a la reforma y $D$ ' a la suma de las distancias entre rentas netas tras la reforma, es decir:

$$
\begin{gathered}
\beta=\frac{\left(1-t^{\prime}\right)-(1-t)}{(1-t)} \\
D=\sum_{i=1}^{N} \sum_{j=1}^{N}\left|\left(x_{i}-t\left(x_{i}\right)\right)-\left(x_{j}-t\left(x_{j}\right)\right)\right|
\end{gathered}
$$

y

$$
D^{\prime}=\sum_{i=1}^{N} \sum_{j=1}^{N}\left|\left(x_{i}-t^{\prime}\left(x_{i}\right)\right)-\left(x_{j}-t^{\prime}\left(x_{j}\right)\right)\right|
$$

De este modo, la ecuación (11) puede expresarse como 


$$
R S^{\prime}-R S=C_{x-t}\left(1-\frac{1}{1+\beta}\right)+\frac{D-D^{\prime}}{2 N^{2} \mu\left(1-t^{\prime}\right)}
$$

Por consiguiente, la variación del índice de ReynoldsSmolenski sería la suma de lo que podríamos llamar un efecto nivel $(E N)$ y un efecto distancia $(E D)$ :

$$
\begin{gathered}
E N=C_{x-t}\left(1-\frac{1}{1+\beta}\right) \\
E D=\frac{D-D^{\prime}}{2 N^{2} \mu\left(1-t^{\prime}\right)}
\end{gathered}
$$

La interpretación de ambos efectos sería la siguiente. El efecto nivel representaría la diferencia entre la curva de concentración de las rentas netas antes de la reforma y la que existiría si dicha reforma se hubiera llevado a cabo mediante una transferencia (positiva o negativa) igual para todos los individuos, de modo que las distancias entre rentas netas hubiesen permanecido constantes. Es importante resaltar que, en nuestro análisis, el "efecto nivel" no está referido al efecto redistributivo de cualquier posible cambio en el impuesto que modifique la recaudación en una determinada cuantía, sino al derivado de una modificación efectuada específicamente mediante una transferencia de cuantía fija para todos los contribuyentes, por lo que difiere del "efecto tipo medio" presente en la descomposición del índice de Reynolds-Smolenski. Así, por ejemplo, para una rebaja impositiva, la representación gráfica de este efecto se expresa en el gráfico 2, donde $L x$-tn representa la curva de concentración tras esa reforma ficticia.

Por su parte, el efecto distancia expresa la diferencia entre la curva de concentración que existiría si la reforma se hubiera realizado mediante transferencias (positivas o negativas) iguales para todos los individuos, manteniendo constantes las distancias entre rentas netas y la curva de concentración posterior a la reforma real $\left(L^{\prime} x-t\right)$ (lo que en rigor implica evaluar los efectos de una reforma puramente redistributiva, que altera las distancias entre las rentas netas en la misma medida que la reforma original analizada, pero sin modificar la recaudación). De este modo, para el caso de una disminución de las distancias, este efecto quedaría también expresado en el gráfico 2 .

La ventaja de esta descomposición del efecto distributivo en distancia y nivel, en comparación con la división tradicional entre nivel y progresividad, radica en que permite identificar claramente si cada
GRÁFICO 2

Reforma fiscal con aumento de la redistribución Efectos nivel y distancia

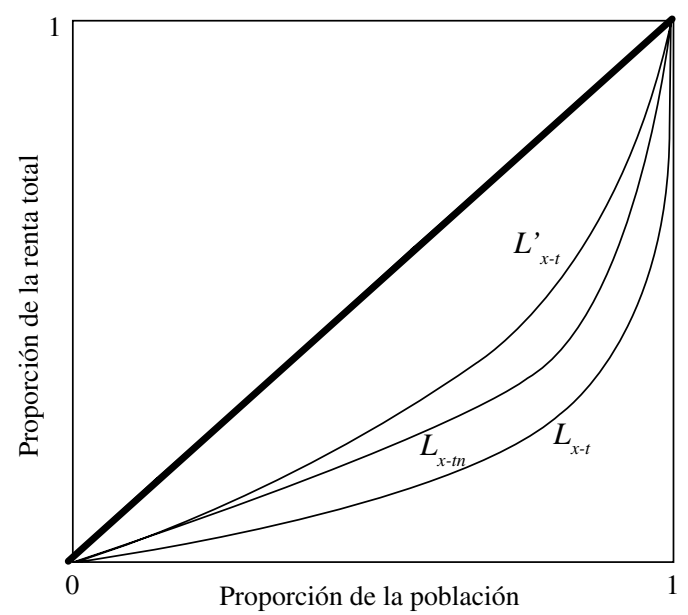

Fuente: elaboración propia.

$L_{x-t}: \quad$ Curva de concentración de la renta después de impuestos previa a la reforma.

$L_{x-t}^{\prime}: \quad$ Curva de concentración de la renta después de impuestos tras la reforma real.

$L_{x-t n}$ : Curva de concentración de la renta tras una reforma de igual efecto recaudatorio realizada con un impuesto de suma fija (en este caso, transferencia).

uno de los dos factores, variaciones en la tasa media y en las distancias, contribuyen positiva o negativamente al cambio generado en la capacidad redistributiva del impuesto, pues compara distancias bajo un hipotético escenario de idéntica recaudación. De esta forma, tanto el efecto nivel como el efecto distancia pueden adoptar signo positivo (contribución positiva a la redistribución) o negativo (contribución negativa). En particular, para el efecto nivel $(E N)$ :

Si $\nabla t \Rightarrow \beta>0 \Rightarrow E N>0$

Si $\Delta t \Rightarrow-1<\beta<0 \Rightarrow E N<0$

Si $\beta=0 \Rightarrow E N=0$

donde $\nabla t$ representa una disminución de los impuestos y $\Delta t$ un aumento de ellos.

Mientras que para el efecto distancia $(E D)$ :

Si $D>D^{\prime} \Rightarrow E D>0$

Si $D<D^{\prime} \Rightarrow E D<0$

Si $D=D^{\prime} \Rightarrow E D=0$

Es importante subrayar que con esta descomposición el efecto de los cambios en el nivel impositivo 
queda perfectamente aislado, lo que no ocurre en la descomposición tradicional. Por ejemplo, si suponemos una reforma fiscal que solo reduce la tasa media, sin modificar las distancias entre cuotas, la descomposición tradicional podría indicar que: i) la capacidad redistributiva del impuesto ha aumentado, ii) la reducción de la tasa media ha contribuido negativamente a ese aumento, y iii) por tanto, el incremento de la progresividad ha sido el único responsable de la mayor capacidad redistributiva.

Sin embargo, lo cierto es que si la progresividad se ha acrecentado, ello obedece únicamente a la reducción de la tasa media y, por consiguiente, esta ha tenido una contribución neta positiva a la capacidad redistributiva, siendo de hecho el único factor causante de su aumento. Por el contrario, la descomposición aquí presentada sí mostraría que la merma de la tasa media tiene un efecto positivo en la capacidad redistributiva del impuesto y que, además, es el único factor responsable de su incremento, mientras que la reforma sería neutral en términos de distancias.

\section{Reformas fiscales y progresividad}

La descomposición anterior aplicada a la redistribución puede también efectuarse sobre la progresividad, empleando para ello el índice de Kakwani (K).

$$
K^{\prime}-K=\left(C^{\prime} t-G^{\prime} x\right)-(C t-G x)
$$

y si de nuevo, para simplificar, suponemos que el coeficiente de Gini antes y después de la reforma no ha cambiado, es decir, $G^{\prime} x=G x$, entonces

$$
\begin{aligned}
& K^{\prime}-K=C^{\prime}{ }_{t}-C_{t}=\frac{\sum_{i=1}^{N} \sum_{j=1}^{N}\left|t^{\prime}\left(x_{i}\right)-t\left(x_{j}^{\prime}\right)\right|}{2 N^{2} \mu t^{\prime}}- \\
& -\frac{\sum_{i=1}^{N} \sum_{j=1}^{N}\left|t\left(x_{i}\right)-t\left(x_{j}\right)\right|}{2 N^{2} \mu t}
\end{aligned}
$$

Esta expresión puede descomponerse del siguiente modo:

$$
\begin{gathered}
K^{\prime}-K=C^{\prime}{ }_{t}-C_{t}=\frac{\sum_{i=1}^{N} \sum_{j=1}^{N}\left|t\left(x_{i}\right)-t\left(x_{j}\right)\right|}{2 N^{2} \mu t}\left(\frac{t}{t^{\prime}}-1\right)+ \\
+\frac{\sum_{i=1}^{N} \sum_{j=1}^{N}\left|t^{\prime}\left(x_{i}\right)-t^{\prime}\left(x_{j}\right)\right|-\sum_{i=1}^{N} \sum_{j=1}^{N}\left|t\left(x_{i}\right)-t\left(x_{j}\right)\right|}{2 N^{2} \mu t^{\prime}}
\end{gathered}
$$

definiendo $\beta$ como la tasa de variación de la tasa impositiva media y $D$ y $D$ ' como la suma de las distancias entre cuotas previas y posteriores a la reforma, se tendrá que:

$$
\begin{gathered}
\beta=\frac{t^{\prime}}{t}-1 \\
D=\sum_{i=1}^{N} \sum_{j=1}^{N}\left|t\left(x_{i}\right)-t\left(x_{j}\right)\right|
\end{gathered}
$$

$\mathrm{y}$

$$
D^{\prime}=\sum_{i=1}^{N} \sum_{j=1}^{N}\left|t^{\prime}\left(x_{i}\right)-t^{\prime}\left(x_{j}\right)\right|
$$

De lo anterior resultaría la siguiente expresión:

$$
K^{\prime}-K=C_{t}\left(\frac{1}{1+\beta}-1\right)+\frac{D-D^{\prime}}{2 N^{2} \mu t^{\prime}}
$$

De este modo, la variación del índice de Kakwani sería la suma de los efectos nivel $(E N)$ y distancia $(E D)$, definidos ahora como

$$
\begin{gathered}
E N=C_{t}\left(\frac{1}{1+\beta}-1\right) \\
E D=\frac{D^{\prime}-D}{2 N^{2} \mu t^{\prime}}
\end{gathered}
$$

En este caso, el efecto nivel representaría la diferencia entre la curva de concentración de las cuotas antes de la reforma y la que se obtendría si esta se hubiera realizado mediante una transferencia (positiva o negativa) igual para todos los individuos, manteniendo las distancias constantes. Por ejemplo, para una rebaja impositiva, la representación gráfica de este efecto se observa en el gráfico 3, donde Ltn representa la curva de concentración de las cuotas con esa reforma ficticia. Por su parte, el efecto distancia expresa la diferencia entre la curva de concentración que existiría si la reforma se hubiera realizado mediante transferencias (positivas o negativas) iguales para todos los individuos, manteniendo constantes las distancias entre cuotas y la curva de concentración posterior a la reforma real $\left(L^{\prime} t\right)$. De esta forma, para el caso de un aumento en las distancias, este efecto quedaría también expresado en el gráfico 3. 
GRÁFICO 3

Efectos nivel y distancia en la progresividad

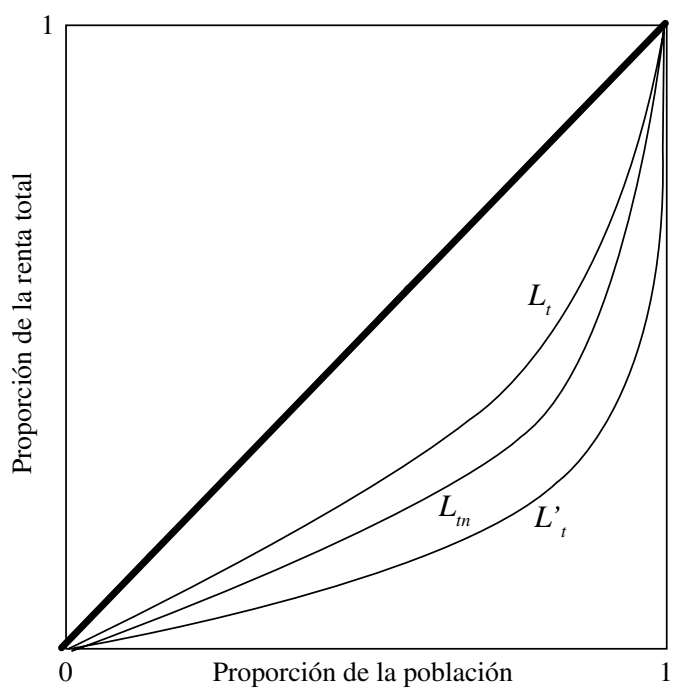

Fuente: elaboración propia.

$L_{t}: \quad$ Curva de concentración de la renta después de impuestos previa a la reforma.

$L_{\text {, } t}$ : Curva de concentración de las cuotas tras la reforma real.

$L_{t n}$ : Curva de concentración de las cuotas tras una reforma de igual efecto recaudatorio realizada con un impuesto de suma fija (en este caso, transferencia).

Nuevamente, la ventaja de esta descomposición es que permite separar el efecto del nivel medio impositivo del correspondiente a las diferencias entre cuotas. Es decir, mientras que el análisis tradicional solo indica si la progresividad ha cambiado, pero no si ello obedece a la variación de la tasa impositiva media o a cambios reales en las diferencias entre cuotas, la descomposición propuesta sí permite efectuar esta distinción. De este modo, tanto el efecto nivel como el efecto distancia pueden adoptar signo positivo (contribución positiva a la progresividad) o negativo (contribución negativa). En particular, para el efecto nivel $(E N)$ :

Si $\Delta t \Rightarrow \beta>0 \Rightarrow E N<0$

Si $\nabla t \Rightarrow<\beta<0 \Rightarrow E N>0$

Si $\beta=0 \Rightarrow E N=0$

donde $\nabla t$ representa una disminución de los impuestos y $\Delta t$ un aumento de ellos.

Mientras que para el efecto distancia $(E D)$ :

Si $D>D^{\prime} \Rightarrow E D<0$

Si $D<D^{\prime} \Rightarrow E D>0$

Si $D=D^{\prime} \Rightarrow E D=0$

\section{Tipología de reformas impositivas}

Una vez definidos los efectos distancia y nivel producidos por una reforma impositiva, pueden clasificarse las distintas modalidades de reformas impositivas en función de estos efectos. Así, en el caso de la capacidad redistributiva del impuesto, la clasificación que se propone es la registrada en el gráfico 4 .

Utilizando esta tipología las reformas impositivas se clasificarían no solo en función de la capacidad redistributiva del impuesto, sino también de lo sucedido con las distancias entre las rentas netas de los contribuyentes. De forma sintética, el siguiente indicador (índice redistributivo distancia-nivel) permite evaluar las diferentes reformas:

$$
I_{R}=\frac{\Delta R S}{|\Delta R S|}\left(1+\frac{E D}{|E D|+|E N|}\right)
$$

$$
\frac{\Delta R S}{|\Delta R S|}= \pm 1 ; 0 \leq\left(1+\frac{E D}{|E D|+|E N|}\right) \leq 2
$$

De este modo, el componente $\frac{\Delta R S}{|\Delta R S|}$ aportaría el signo del indicador y $\left(1+\frac{E D}{|E D|+|E N|}\right)$ su valor absoluto, que recogería la importancia relativa del efecto distancia en la reforma. En consecuencia, de acuerdo con este indicador, las reformas se clasificarían en:

i) Reforma redistributiva fuerte, si $1<I_{R} \leq 2(\triangle R S$, $E D>0$ ).

GRÁFICO 4

\section{Redistribución. Tipos de reforma impositiva}

Tipos de
reforma $\left\{\begin{array}{l}\Delta R S\left\{\begin{array}{l}E D>0 \text { Reforma redistributiva fuerte } \\ E D<0 \text { Reforma redistributiva débil }\end{array}\right. \\ \nabla R S\left\{\begin{array}{l}E D>0 \text { Reforma no redistributiva débil } \\ E D<0 \text { Reforma no redistributiva fuerte }\end{array}\right.\end{array}\right.$

Fuente: elaboración propia.

RS: Índice de Reynolds-Smolensky.

$E D$ : Efecto distancia. 
ii) Reforma redistributiva débil, si $0<\mathrm{I}_{\mathrm{R}} \leq 1(\Delta R S$, $E D<0)$.

iii) Reforma no redistributiva débil, si $-2 \leq \mathrm{I}_{\mathrm{R}}<-1$ $(\nabla R S, E D>0)$.

iv) Reforma no redistributiva fuerte, si $-1 \leq \mathrm{I}_{\mathrm{R}} \leq 0$ $(\nabla R S, E D<0)$.

En el caso de la progresividad, el análisis es similar. De nuevo existirían cuatro tipos posibles de reformas en función de la variación del índice de Kakwani y del signo del efecto distancia, siendo el indicador (índice de progresividad distancia-nivel) correspondiente:

$$
\begin{gathered}
I_{K}=\frac{\Delta K}{|\Delta K|}\left(1+\frac{E D}{|E D|+|E N|}\right) \\
\frac{\Delta K}{|\Delta K|}= \pm 1 ; 0 \leq\left(1+\frac{E D}{|E D|+|E N|}\right) \leq 2
\end{gathered}
$$

Su significado e interpretación sigue los mismos patrones, pero en este caso aplicados a la progresividad:

i) Reforma progresiva fuerte, si $1<I_{K} \leq 2(\Delta K$, $E D>0$ )

ii) Reforma progresiva débil, si $0<I_{K} \leq 1(\Delta K$, $E D<0)$

iii) Reforma regresiva débil, si $-2 \leq I_{K}<-1$ ( $\nabla K$, $E D>0)$

iv) Reforma regresiva fuerte, si $-1 \leq I_{K} \leq 0$ ( $\nabla K$, $E D<0)$

En definitiva, con estos indicadores la clasificación de las reformas fiscales se enriquece, añadiendo a los calificativos tradicionales de "redistributiva" o "progresiva" (basados en el valor positivo o negativo de $R S$ y $K$ ) -que se mantienen conservando la coherencia con los indicadores clásicos- el carácter de "fuerte" o "débil" derivado de la contribución que realice en cada caso el efecto distancia. Además, en cuanto el valor de los índices se encuentre normalizado con respecto a la cuantía de los efectos recaudatorios, será posible comparar entre reformas fiscales con diferente efecto cuantitativo.

\section{Reforma fiscal y elementos del impuesto: efectos sobre nivel y distancia}

La descomposición de las variaciones en los indicadores de redistribución y progresividad realizada en las subsecciones anteriores permite evaluar los efectos de las reformas fiscales, distinguiendo entre la cuantía de la rebaja fiscal y los efectos de los diferentes elementos del impuesto utilizados para llevarla a cabo. De este modo, el efecto nivel permite aislar el componente puro de aumento o disminución de los impuestos, siendo dicho efecto el mismo para todas las reformas fiscales que afecten de igual forma a la recaudación. Por tanto, este procedimiento permite observar las diferencias entre las diversas reformas posibles que impliquen la misma variación recaudatoria, diferencias que aparecerán recogidas en el efecto distancia. Así, a modo de ejemplo, en el cuadro 1 se sintetizan los efectos de tres posibles medidas de reforma de un impuesto sobre la renta cuya tarifa previa fuese progresiva.

El efecto distancia por separado mide una variación en la distribución (o en la progresividad) con valoración normativa, ya que compara dos distribuciones de renta (o dos estructuras impositivas) por su desviación con respecto a la proporcionalidad, manteniendo constante la renta neta media (o la recaudación). Puesto que, dada la cuantía de una rebaja fiscal, el efecto nivel es constante, se puede valorar normativamente el diseño (los instrumentos utilizados) de la reforma. En relación con los ejemplos indicados en el cuadro 1, el

CUADRO 1

\section{Efectos distancia y nivel en reformas alternativas}

1. Deducciones en cuota

Introducción o aumento de una reducción en cuota igual para todos los contribuyentes

\begin{tabular}{lll} 
Progresividad & $\mathrm{EN}>0$ & \\
& $\mathrm{ED}=0$ & Aumento de K \\
Redistribución & $\mathrm{EN}>0$ & \\
& $\mathrm{ED}=0$ & \\
& & \\
\hline
\end{tabular}

2. Reducciones en base

Introducción o incremento de una reducción de igual cuantía para todos los contribuyentes

$\begin{array}{lll}\text { Progresividad } & \text { EN }>0 & \\ & \text { ED }<0 & \text { K } \\ \text { Redistribución } & \text { EN }>0 & \\ & \text { ED }<0 & \text { RS }\end{array}$

3. Reducción de tasas impositivas

Reducción de tasas marginales en la tarifa (independientemente de cuáles se rebajen)

$\begin{array}{lll}\text { Progresividad } & \text { EN }>0 & \\ & \text { ED }<0 & \text { K } \\ \text { Redistribución } & \text { EN }>0 & \\ & \text { ED }<0 & \text { RS }\end{array}$

Fuente: elaboración propia.

$E N$ : Efecto nivel.

$E D$ : Efecto distancia.

$R S$ : Índice de Reynolds-Smolensky.

$K$ : Índice de Kakwani. 
diseño de la reforma 1 (deducciones en cuota) sería neutral en términos de aportación a la progresividad y redistribución $(E D=0)$, mientras que el diseño de las reformas 2 y 3 (reducciones en base y tasas impositivas) sería perjudicial (disminuiría) en términos de progresividad y capacidad redistributiva del impuesto $(E D<0)$. La valoración de otras alternativas de reforma (deducciones o reducciones de cuantía variable por niveles de renta; modificación de los tramos de la tarifa; combinación de aumentos y disminuciones en las deducciones, reducciones o tarifas; cambios en la determinación de la renta sometida a gravamen, entre otras), así como de los efectos conjuntos de la combinación de diferentes medidas, es más compleja, debiendo procederse — en su caso- al cálculo de los indicadores correspondientes.

\section{$\mathrm{V}$}

\section{Una aplicación al impuesto sobre la renta personal de Guatemala}

Pese a los esfuerzos realizados en la última década en materia de administración y diseño de la política fiscal, la recaudación tributaria en Guatemala sigue siendo una de las más bajas de América Latina. ${ }^{7}$ Aunque las tasas nominales de los impuestos no son demasiado diferentes de la media latinoamericana, la alta informalidad, la desigual distribución de la renta, la estrechez de las bases imponibles y el elevado fraude explican estas insuficiencias recaudatorias. En cuanto a la estructura de la tributación, Guatemala sobresale por el reducido peso de la imposición sobre la renta personal, ${ }^{8}$ mientras que la participación de la imposición sobre sociedades y sobre el consumo es relativamente elevada (véase el cuadro 2 ).

Si se analiza en mayor detalle la tributación por fuentes de renta, se observa que los salarios (impuesto sobre la renta personal en régimen de dependencia) apenas contribuyen a la recaudación: $0,13 \%$ del producto interno bruto (PIB), lo que supone el 3,92\% de la tributación global de las rentas. Este hecho contrasta con la participación de dichos salarios en el PIB, que - según datos de Contabilidad Nacional- ascendía al $32 \%$ en 2006. Es decir, solo se estaría recaudando un $0,34 \%$ de la masa salarial, una ratio excesivamente baja cuyas causas van más allá de la elevada informalidad y la extrema desigualdad existente en Guatemala. Entre

\footnotetext{
${ }^{7}$ Cabe destacar que entre 2003 y 2008, la presión tributaria de América Latina y el Caribe se incrementó de un $15,5 \%$ a un $17,8 \%$ del PIB, mientras que, en contraste, la correspondiente a Guatemala disminuyó de un $11,9 \%$ a un $11,6 \%$ del PIB (CEPAL, 2009).

${ }^{8}$ En línea, en todo caso, con la mayoría de los sistemas fiscales en América Latina cuyas reformas han sido parciales, subsistiendo una débil tributación directa.
}

\section{CUADRO 2 \\ Guatemala: ingresos tributarios en 2006 \\ (En porcentajes del PIB)}

\begin{tabular}{lr}
\hline Impuestos directos & 2,9 \\
\hline Impuesto sobre la renta & 2,1 \\
de personas jurídicas & 1,7 \\
de personas individuales & 0,3 \\
Impuesto sobre productos financieros (personas jurídicas) & 0,1 \\
Impuesto sobre productos financieros (personas individuales) & 0,0 \\
IETAAPa & 0,8 \\
Impuestos sobre el patrimonio & 0,0 \\
\hline Impuestos indirectos & 7,7 \\
\hline Impuesto al valor agregado doméstico & 1,9 \\
Impuesto al valor agregado sobre importaciones & 3,4 \\
Derechos arancelarios & 1,0 \\
Impuesto sobre tabaco y sus productos & 0,1 \\
Impuestos sobre distribución de bebidas & 0,2 \\
Impuesto sobre circulación de vehículos & 0,1 \\
Impuesto sobre distribución de petróleo y derivados & 0,7 \\
Total de ingresos tributarios & 11,1 \\
\hline
\end{tabular}

Fuente: Superintendencia de Administración Tributaria (SAT).

a Impuesto extraordinario y temporal de apoyo a los Acuerdos de Paz.

PIB: Producto interno bruto.

las explicaciones de esta insuficiencia recaudatoria se encuentra la legislación sobre los ingresos computables y los gastos deducibles en el cómputo del rendimiento sujeto a gravamen. Por una parte, no se tributa por rentas como aguinaldos, Bono 14, indemnizaciones por tiempo de servicio, pensiones o jubilaciones y montepíos. Por otra, se permite la deducción de primas de seguro de vida, ciertas donaciones, gastos médicos e, incluso, el 
crédito fiscal por el IVA pagado en las compras hasta el $12 \%$ de la renta neta. Por último, existe una deducción en la base de 36.000 quetzales, para efectos de mínimo vital y umbral de tributación, que si bien no es demasiado elevada en el contexto centroamericano (2,03 veces el PIB per cápita de 2006), contribuye a la pérdida de recaudación y de progresividad.

En cuanto a la imposición sobre el capital en el ámbito personal, esta se encuentra dispersa pese a guardar una estructura claramente dual: las plusvalías tributan en el impuesto sobre la renta a una tasa del 10\%, los intereses y similares tributan en el impuesto sobre productos financieros a la misma tasa del 10\%, mientras que los dividendos se encuentran exentos siempre que hayan tributado en la fuente.

A fin de analizar el impuesto sobre la renta en Guatemala en relación con las personas individuales en régimen de dependencia, dado que no se dispone de información desagregada sobre las rentas sujetas y los impuestos pagados, se ha recurrido en el presente análisis a los datos de la Encuesta Nacional de Empleo e Ingresos (ENEI) 2004. Para ello, se ha procedido a adaptar dicha base de datos a la estructura del impuesto, realizando hipótesis respecto de las variables básicas no contempladas en la encuesta a objeto de aproximar el escenario base (legislación de 2006) a los resultados de recaudación reales. 9

Esta réplica del escenario base de 2006 muestra un impuesto de baja recaudación, concentrado en un número muy pequeño de contribuyentes efectivos y con escasa capacidad redistributiva, pese a su elevada progresividad formal. El nivel del mínimo exento es el primer responsable de esta baja tributación media. Así, aunque existen cuatro tasas marginales: 15\%, 20\%, 25\% y $31 \%$ (véase el cuadro 3), deja exentos de tributación al $73 \%$ de los asalariados, aplicándose la tasa máxima

\footnotetext{
${ }^{9}$ Las rentas declaradas en la encuesta se han agrupado en función de sus categorías de rentas salariales sujetas, rentas salariales exentas, pensiones, intereses, dividendos, alquileres, plusvalías y rentas de actividades agrícolas y no agrícolas. Estas rentas han sido actualizadas a valores de 2006 mediante el índice de precios al consumidor, excepto intereses y dividendos, en cuyo caso se ha imputado a los perceptores de dichas rentas la parte proporcional que les correspondería de los totales de dividendos e intereses percibidos por los hogares de acuerdo con las cifras de Contabilidad Nacional. Para el cálculo del escenario base de igual recaudación a la real, se ha estimado que la renta neta gastada con justificación de cara a la aplicación del crédito por IVA es del $45 \%$. Por otra parte, se ha excluido de la base a los individuos con edad inferior a la mínima para trabajar y se han clasificado como "informales" todos aquellos carentes de contrato de trabajo. Finalmente, el tratamiento de los datos es a nivel individual (más adecuado para efectos fiscales), no por hogares, lo que ha de tenerse en cuenta a la hora de interpretar los valores de los indicadores de desigualdad.
}

CUADRO 3

Guatemala: escala de tasas del impuesto sobre la renta (personas), 2006

\begin{tabular}{lcc}
\hline $\begin{array}{l}\text { Tramos } \\
\text { (en quetzales) }\end{array}$ & $\begin{array}{c}\text { Tasa marginal } \\
\text { (en porcentajes) }\end{array}$ & Percentiles \\
\hline Hasta $36000^{\mathrm{a}}$ & 0 & $1-73(73 \%)$ \\
$36000-65000$ & 15 & $74-92(19 \%)$ \\
$65000-180000$ & 20 & $93-99(7 \%)$ \\
$180000-295000$ & 25 & $100(1 \%)$ \\
295000 en adelante & 31 & \\
\hline
\end{tabular}

Fuente: elaboración propia.

a Esta cuantía se resta de la renta sujeta a tributación.

a rentas muy elevadas (16,7 veces el PIB per cápita). Todo ello con Figura una escala de tasas excesivamente compleja para su escaso recorrido en términos de renta e individuos afectados por ella.

La existencia del crédito por IVA acentúa esta tendencia, ya que tras su aplicación únicamente pagan el impuesto el 9,65\% de los trabajadores formales, todos ellos en el último decil de la clasificación. Además, el crédito por IVA reduce el impuesto pagado en el $62 \%$. Este efecto conjunto del mínimo exento y del crédito por IVA es tan fuerte que, de hecho, la eliminación de la tasa impositiva del $31 \%$ no provocaría, en la simulación efectuada, ninguna variación en los resultados (ningún contribuyente se vería afectado). Como consecuencia de todo ello, los indicadores de progresividad y redistribución denotan un impuesto muy progresivo (Kakwani $=0,6136$ ), pero muy poco redistributivo (Reynolds-Smolensky $=0,0072$ ), debido a su escasa capacidad recaudatoria (la tasa media efectiva es del 1,34\%).

En vista de todo ello y a objeto de poner de manifiesto las limitaciones de los indicadores tradicionales de redistribución y progresividad, en este trabajo se han planteado dos reformas ficticias que inciden en estos dos elementos del impuesto. En primer lugar, la modificación del mínimo exento. $\mathrm{Al}$ respecto, conviene subrayar que su existencia obedece al menos a tres objetivos básicos: ${ }^{10}$ i) establecer el umbral de renta necesaria para configurar un mínimo vital, dejando exentas las rentas inferiores a este nivel; ii) disminuir la presión tributaria en función de la capacidad económica de los contribuyentes, teniendo en cuenta sus circunstancias familiares y personales; y iii) simplificar el impuesto, tanto para la administración como para los

\footnotetext{
${ }^{10}$ Véase, por ejemplo, Zee (2005).
} 
ciudadanos, evitando su pago por parte de los numerosos contribuyentes de renta muy reducida.

A la hora de establecer esta renta mínima exenta de tributación, las reducciones en la base (como la vigente en Guatemala), los tramos a tasa cero o los créditos fiscales pueden ser equivalentes, pero el costo recaudatorio es mucho más elevado en el caso de las reducciones y la visibilidad es menor en el caso de los créditos. Además, debido a que el ahorro impositivo de cada contribuyente se produce a su tasa marginal máxima, dicho ahorro es creciente en función de la renta. Por ello, a nivel internacional se está produciendo una tendencia a sustituir las reducciones en la base por tramos tasa cero o créditos (OCDE, 2006).

Teniendo en cuenta todo lo anterior, se ha planteado aquí su transformación en un tramo a tasa cero de igual cuantía que la actual. En ese caso, la recaudación aumentaría un $21 \%$, mientras que se produciría un incremento del índice de Reynolds-Smolensky y una caída del índice de Kakwani, de modo que la interpretación de la reforma basada en estos indicadores mostraría un crecimiento de la capacidad redistributiva, pero una reducción de la progresividad, por lo que dicho aumento sería tan solo consecuencia de la mayor tasa media (véase el cuadro 4). Por tanto, una reforma de este tipo podría ser criticada con el argumento de que la ganancia en la capacidad redistributiva del impuesto es únicamente fruto de la mayor tasa media, pero que en realidad se está configurando un impuesto menos progresivo. Pues bien, como evidencian los índices aquí propuestos, lo que sucede en realidad es que esta caída de la progresividad, medida por el índice de Kakwani, es también producto del aumento de la

CUADRO 4

Transformación del mínimo personal a tramo tasa cero

\begin{tabular}{|c|c|c|c|}
\hline & Post reforma & Pre reforma & Variación \\
\hline Recaudación (en quetzales) & 354348097 & 291670901 & $21 \%$ \\
\hline$R S$ & 0,0090 & 0,0072 & 0,0018 \\
\hline \multirow[t]{2}{*}{$K$} & 0,6119 & 0,6136 & $-0,0017$ \\
\hline & RS & K & \\
\hline Efecto nivel & $-0,0010$ & $-0,1694$ & \\
\hline Efecto distancia & 0,0028 & 0,1677 & \\
\hline$I_{R}$ & 1,7381 & & \\
\hline$I_{K}^{K}$ & & $-1,4975$ & \\
\hline
\end{tabular}

\section{Fuente: elaboración propia.}

$R S:$ Índice de Reynolds-Smolensky.

$K$ : Índice de Kakwani.

$I_{R}:$ Índice redistributivo distancia-nivel.

$I_{K}$ : Índice de progresividad distancia-nivel. tasa media. El efecto nivel, relacionado con esta última, es el que contribuye en ambos casos — progresividad y capacidad redistributiva - de forma negativa. Por el contrario, el efecto distancia lo hace de manera positiva, lo que implica que no solo se reducen significativamente las distancias entre las rentas netas, sino que también se acrecientan las diferencias entre las cuotas que pagan los contribuyentes. Por ello, difícilmente puede decirse que la reforma es regresiva y, de serlo, se perdería la percepción subjetiva que el ciudadano tiene sobre la progresividad. Por el contrario, en la propuesta de este trabajo se define una reforma como la simulada, separando lo ocurrido con la tasa media de lo sucedido con las distancias entre rentas y cuotas. En este ejemplo concreto, en que ha aumentado la diferencia entre los impuestos pagados por un individuo de renta alta y por otro de renta baja, los indicadores propuestos señalarían que se trata de una reforma redistributiva fuerte $\left(I_{R}=1,74\right)$ y regresiva débil $\left(I_{K}=-1,50\right)$.

En segundo lugar, se ha simulado una reforma en que se considera la eliminación del crédito por IVA, lo que supondría un incremento relevante de la recaudación (164\%). Respecto de sus consecuencias en la capacidad redistributiva y la progresividad del impuesto, sucedería lo mismo que en la medida anterior: una aparente pérdida de progresividad, producto solamente de la mayor tasa media (efecto nivel negativo) (véase el cuadro 5). Por el contrario, aumentarían las diferencias entre los impuestos pagados por los individuos de rentas baja y alta (efecto distancia positivo). En definitiva, se trataría nuevamente de una reforma redistributiva fuerte $\left(I_{R}=1,72\right)$ y regresiva débil $\left(I_{K}=-1,47\right)$.

CUADRO 5

\section{Eliminación del crédito por IVA}

\begin{tabular}{|c|c|c|c|}
\hline & Post reforma & Pre reforma & Variación \\
\hline Recaudación (en quetzales) & 769134255 & 291670901 & $164 \%$ \\
\hline$R S$ & 0,0189 & 0,0072 & 0,0118 \\
\hline \multirow[t]{2}{*}{$K$} & 0,5502 & 0,6136 & $-0,0634$ \\
\hline & RS & K & \\
\hline Efecto nivel & $-0,0076$ & $-0,5944$ & \\
\hline Efecto distancia & 0,0194 & 0,5310 & \\
\hline$I_{R}$ & 1,7178 & & \\
\hline$I_{K}$ & & $-1,4718$ & \\
\hline
\end{tabular}

Fuente: elaboración propia.

$R S$ : Índice de Reynolds-Smolensky.

$K$ : Índice de Kakwani.

$I_{R}$ Índice redistributivo distancia-nivel.

$I_{K}$ : Índice de progresividad distancia-nivel.

IVA: Impuesto al valor agregado. 


\section{VI}

\section{Conclusiones}

A lo largo del presente trabajo se ha tratado de poner de manifiesto las limitaciones de los índices de Kakwani y de Reynolds-Smolensky para analizar los efectos en la progresividad y capacidad redistributiva de los impuestos cuando se pretende analizar reformas fiscales que implican cambios en la recaudación. Asimismo, se ha indicado que resultan discutibles las formas tradicionales de solventar dichas insuficiencias mediante el uso de curvas generalizadas y la descomposición del índice de Reynolds-Smolensky en los efectos causados por la tasa media y por la progresividad. En el primer caso, por sesgar injustificadamente los resultados en favor de las reducciones de impuestos. En el segundo, porque descomponer los cambios del índice de ReynoldsSmolensky en los efectos causados por la tasa media y por la progresividad no es posible para los efectos valorativos del diseño de las reformas, pues la propia medición de la progresividad se ve alterada en la mayoría de las reformas reales debido a los mismos factores que alteran la tasa media de los impuestos. En consecuencia, se han planteado unos indicadores que permiten cuantificar los efectos de una reforma impositiva con cambios en la recaudación a partir de dos conceptos que sí son separables: el nivel impositivo y las diferencias entre rentas netas o cuotas. Estos indicadores permiten distinguir los efectos tanto en la capacidad redistributiva como en la progresividad del impuesto causados por los cambios en la tasa media y por las variaciones de las diferencias de cuotas impositivas entre contribuyentes.

Por otra parte, los efectos nivel y distancia desarrollados de este modo permiten recuperar en parte el sentido intuitivo de los conceptos de progresividad y redistribución. Determinar "a quién beneficia más" una reforma fiscal es complicado y está sujeto a juicios de valor. Los indicadores tradicionales $(K, R S, \mathrm{y}$ su descomposición) aportan una visión basada en las diferencias relativas de renta o cuotas muy útil a objeto de realizar comparaciones en un contexto estático para reformas que no producen cambios en la recaudación tributaria. En caso contrario, las conclusiones que se obtienen pueden resultar contraintuitivas. Por ejemplo, ¿cómo puede aumentar la progresividad del impuesto una reforma que rebaje mucho más las cuotas de los individuos de renta alta, siendo estos quienes reciben la mayor parte de la rebaja fiscal tanto en términos absolutos como relativos? Si esto es cierto, ¿es "bueno" aumentar la progresividad? O visto de otro modo, si estuvieran bien informados, ¿votaría la mayoría de los ciudadanos en favor de una reforma de este tipo?

Como se ha intentado demostrar en el presente trabajo, la confusión parte de la interpretación de los indicadores utilizados para la valoración de las reformas fiscales cuando varía la recaudación. En este trabajo se propone una opción diferente. El efecto nivel aísla las repercusiones que una reforma tendría en los porcentajes de renta o cuotas impositivas soportados por los contribuyentes (en definitiva, en los indicadores de progresividad y redistribución), en caso de que las distancias entre cuotas y rentas permaneciesen constantes. El efecto distancia recoge las consecuencias del diseño concreto de la reforma (esto es, de los elementos del impuesto modificados) en la progresividad y la redistribución, cuando el nivel de recaudación y de renta total permanece constante. Esta descomposición de los efectos, así como los indicadores construidos a partir de ella, permiten matizar y enriquecer las conclusiones alcanzadas mediante el uso de los indicadores clásicos.

Además, esta descomposición alternativa de los efectos de una reforma impositiva permite, sin dejar de utilizar los instrumentos tradicionales basados en una concepción relativa de la desigualdad, resaltar el efecto de la variación de las distancias entre rentas netas o entre cuotas, lo que a nuestro juicio tiene un doble interés. Por una parte, de cara a la explicación de las repercusiones de una reforma fiscal, los gestores y ciudadanos pueden estar interesados en conocer sus consecuencias en términos absolutos y qué efectos se producen en la distancia entre las rentas (o cuotas) de los individuos. Por otra, esta descomposición permite una aproximación a las "hipótesis de renta relativa". Aunque no puede considerarse en puridad que los indicadores descritos en este trabajo recogen el efecto en la posición relativa de los contribuyentes en un escenario de reforma fiscal, sí ponen de manifiesto su efecto agregado en el conjunto de posiciones relativas a través del cómputo de la variación en las distancias entre rentas o cuotas.

Finalmente, conviene subrayar que los problemas de los indicadores tradicionales evidenciados a lo largo de estas páginas resultan de mayor gravedad en el caso de países de baja tributación. A menudo, una reforma que genere el necesario incremento de la capacidad recaudatoria del sistema fiscal aparecerá, de acuerdo con dichos 
indicadores, como regresiva, lo que puede ser empleado como argumento para su rechazo. Sin embargo, esta aparente regresividad es solo consecuencia del propio aumento de la capacidad recaudatoria y no de un acercamiento entre las cuotas pagadas por los contribuyentes de rentas alta y baja. Como se ha podido comprobar en las medidas tributarias analizadas en el caso de Guatemala, los indicadores habituales señalarían que la progresividad del impuesto ha disminuido. Sin embargo, lo cierto es que en ambos casos aumentan las diferencias entre los impuestos pagados por los individuos de mayor y menor nivel de ingreso, lo que parece contradictorio con lo anterior. Por el contrario, en la presente propuesta se deja ver claramente que dicha reducción de la progresividad es solo consecuencia de la mayor tasa media tras las reformas, pero que el costo de ellas afectará más a los contribuyentes de mayor ingreso. A nuestro juicio, se trata de una información de notable interés social.

\section{Bibliografía}

Atkinson, A.B. (1970), "On the measurement of inequality", Journal of Economic Theory, vol. 2, $\mathrm{N}^{\circ} 3$, Amsterdam, Elsevier.

CEPAL (Comisión Económica para América Latina y el Caribe) (2009), Estudio económico de América Latina y el Caribe, 2008-2009 (LC/G.2410-P), cuadro A-37, Santiago de Chile. Publicación de las Naciones Unidas, $\mathrm{N}^{\mathrm{o}}$ de venta: S.09.II.G.2.

Kakwani, N.C. (1977), "Measurement of tax progressivity: an international comparison", The Economic Journal, vol. 87, $\mathrm{N}^{\mathrm{o}} 345$, Londres, Royal Economic Society.

Lambert, P. (2001), The Distribution and Redistribution of Income, Manchester, Manchester University Press.
OCDE (Organización de Cooperación y Desarrollo Económicos) (2006), "Fundamental reform of personal income tax", Tax Policy Studies, No 13, París.

Reynolds, M. y E. Smolensky (1977), Public Expenditures, Taxes and the Distribution of Income: The United States, 1950, 1961, 1970, Nueva York, Academic Press.

Shorrocks, A.F. (1983), "Ranking income distributions", Economica, vol. 50, $\mathrm{N}^{\mathrm{o}}$ 197, Londres, London School of Economics and Political Science.

Zee, H.H. (2005), "Personal income tax reform: concepts, issues, and comparative country developments", IMF Working Papers, $\mathrm{N}^{\circ} 87$, Washington, D.C, Fondo Monetario Internacional. 